\title{
DNA-Binding and Fluorescence Properties OF THE DNA BIS-INTERCALATING Purple Oxazole Dimer Popo-1
}

\author{
Stefan Winter and Günter Löber \\ Institut für Molekulare Biotechnologie e.V., Beutenbergstr. 11, D-07745 Jena, Germany \\ (Paper JBO-063 received Dec. 8, 1995; revised manuscript received Nov. 4, 1996; accepted for publication Nov. 12, 1996.)
}

\begin{abstract}
Dimers of the fluorescent DNA intercalators oxazole yellow and thiazole orange are used for high-sensitivity DNA detection due to their excellent fluorescence properties. Fluorescence lifetime techniques and absorption spectroscopy were used to investigate the DNA binding properties of POPO-1 [4,4,8,8-tetramethyl-4,8diazaundecamethylene)bis-4-(3-methyl-2,3-dihydrobenzo-1,3-oxazolyl)-2-methylidene] with the doublestranded homopurine-homopyrimidine polynucleotides poly $(\mathrm{dA} \cdot \mathrm{dT})$, poly $(\mathrm{dG} \cdot \mathrm{dC})$ and calf thymus DNA. The coexistence of different binding modes of POPO- 1 with polynucleotides such as bisintercalation and monointercalation was found in connection with minor groove binding as well as electrostatic attachment. At high excess of polynucleotides, bisintercalation is the only existing form of binding whereas an increasing amount of POPO-1 leads to the coexistence of bis- and monointercalated dye molecules. The amount of bound dye increases with decreasing ionic strength of the buffer and is dependent on the polynucleotide itself. The best binding conditions were found with calf thymus DNA, followed by poly (dA.dT) and poly(dG·dC). ๑ 1997 Society of Photo-Optical Instrumentation Engineers.
\end{abstract}

Keywords fluorescence lifetime; purple oxazole dimer; benzo-oxazoles, bis-intercalation; polynucleotides; DNA.

\section{INTRODUCTION}

In 1992 Rye et al. ${ }^{1}$ described new DNA stains for high-sensitivity DNA detection in gel electrophoresis. Those dyes are dimers of the well-known fluorescent DNA intercalators like oxazole yellow (YO) and thiazole orange (TO). The two fluorophors of such dimers are linked by a biscationic, aliphatic linker, as can be seen in the molecular structure of POPO-1 [4,4,8,8-tetramethyl-4,8-diazaundecamethylene)-bis-4-(3-methyl-2, 3-dihydrobenzo-1, 3-oxazolyl)- 2-methylidene], which is given in Figure 1. The length of the linker is adjusted in such a way as to allow the dimer to bisintercalate into polynucleotides. It was first shown by Gaugin et al. ${ }^{2}$ for the dimer of ethidium that the binding constants of bisintercalated molecules are about 1000 times higher than those of the corresponding monomers. For YOYO (a dimer of oxazole yellow) and TOTO (a dimer of thiazole orange), binding constants are expected to be in the range of $10^{10} M^{-1}$ to $10^{12} M^{-1}$. 3.4

Such compounds are promising candidates for clinical use as potentially antitumor and antiviral drugs. Owing to the stable complexes formed between dye and DNA, the enzyme activity in the DNA replication process can be inhibited. ${ }^{5}$ It was shown recently ${ }^{6}$ that YOYO and TOTO are able to photocleave double-stranded as well as single-

Address all correspondence to Stefan Winter. E-mail: swinter@imbjena.de stranded DNA, depending on their binding modes to DNA.

The dimers mentioned show excellent fluorescence properties. They are very weakly fluorescent in aqueous solution but upon intercalation into polynucleotides, their fluorescence intensity is enhanced by a factor of several hundreds up to a maximum of 3000 . Owing to the resulting low background signal, picograms of DNA fragments can be detected in gels by laser scanning devices. ${ }^{7}$

In our study we investigated the binding properties of the blue-fluorescing dye POPO-1 with calf thymus DNA (ctDNA) and the homopurine/ homopyrimidine double-stranded polynucleotides poly $(\mathrm{dA} \cdot \mathrm{dT})$ and poly $(\mathrm{dG} \cdot \mathrm{dC})$ in buffers of different ionic strength and at molar mixing ratios of dye to DNA base pairs $(\mathrm{d} / \mathrm{bp})$ ranging between 0.005 and 1 . We applied frequency domain fluorescence lifetime techniques as well as absorption and fluorescence spectroscopic investigations.

\section{MATerials AND MeTHOdS}

\subsection{CHEMICALS AND SAMPLE PREPARATION}

Calf thymus DNA (ctDNA), polydeoxyadenylicpolydeoxythymidylic acid [poly(dA.dT)] and polydeoxyguanylic-polydeoxycytidylic acid [poly $(\mathrm{dG} \cdot \mathrm{dC})]$ were purchased from Sigma-Aldrich 


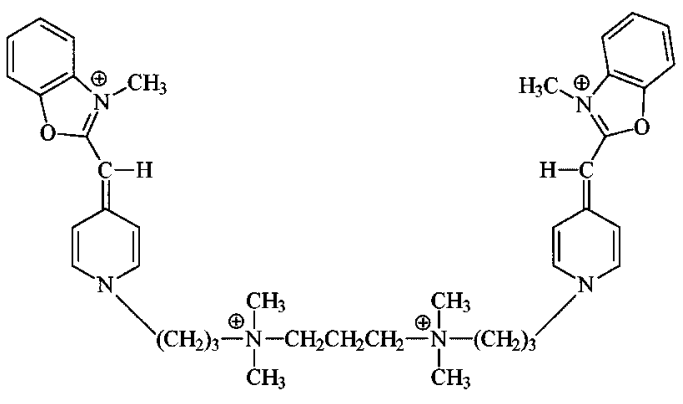

Fig. 1 Molecular structure of the dye POPO-1 used in the investigations.

Chemie GmbH (Deisenhofen, Germany) and used without further purification. In order to obtain smaller fragments of ctDNA, the ctDNA was sonicated for $15 \mathrm{~min}$ in an ultrasonic bath. The length distribution of the fragments was checked by gel electrophoresis, which showed a smeared DNA distribution in the corresponding lane. Maximum concentration was obtained for fragments of 1 to $3 \mathrm{~kb}$ length, as could be seen in comparison with a DNA molecular weight standard.

POPO-1 was purchased from Molecular Probes Inc. (Eugene, Oregon). For titration the 1-mM stock solution was diluted by a 1:4 dimethyl sulfoxide (DMSO)-water mixture to 0.375 and $0.0375 \mathrm{mM}$ working solutions. Two buffers of different ionic strength were prepared in the following compositions: buffer 1: $0.15 \mathrm{mM} \mathrm{NaCl}, 10 \mathrm{mM}$ tris, $\mathrm{pH}$ 7.4; buffer 2: $0.015 \mathrm{mM} \mathrm{NaCl}, 10 \mathrm{mM}$ tris, $\mathrm{pH}$ 7.4.

Poly $(\mathrm{dA} \cdot \mathrm{dT})$, poly $(\mathrm{dG} \cdot \mathrm{dC})$ and ctDNA were dissolved in buffer 1 and buffer 2 . The concentration of the polynucleotides was adjusted to $15 \mu \mathrm{mol} /$ liter by absorption measurements, taking into account the different molar extinction coefficients of the polynucleotides. In order to avoid precipitation of the DNA at large excess of POPO-1, $500 \mu$ l of buffer containing $7.5 \mathrm{nM}$ of the corresponding polynucleotide were titrated with the dye by stepwise addition of the dye working solutions. The relative amount of POPO-1 per base pair is given by the mixing ratio $d / b p$, which was varied between $d / b p$ $=0.005$ and $\mathrm{d} / \mathrm{bp}=1.0$. After each addition of aliquotes of POPO-1, the solution was stirred for 2 min before starting absorption and fluorescence measurements. Owing to the high binding affinity of POPO- 1 to the DNA, this time should be sufficient to achieve equilibrium.

\subsection{METHODS AND INSTRUMENTATION}

Absorption spectra were recorded in the visible region on a Cary 4E spectrophotometer (Varian Ltd. Pty., Mulgrave, Australia). The estimated molar extinction coefficient of free POPO-1 amounts to $\epsilon=107,000 \mathrm{M}^{-1} \mathrm{~cm}^{-1}$ at $410 \mathrm{~nm}$.

The circular dichroism spectra of the polynucleotides were obtained with a Jasco J-710 spectropolarimeter (Japan Spectroscopic Co., Ltd., Tokyo, Ja- pan) in a 1-mm silica cuvette. Fluorescence emission spectra were measured with a KontronSFM25 spectrofluorimeter (Tegimenta AG, Zurich, Switzerland) between 440 and $600 \mathrm{~nm}$. Owing to the lack of changes in the emission maximum during titration, the peak height at maximum emission $(456 \mathrm{~nm})$ was used to characterize the fluorescence intensity at each titration step. Fluorescence lifetimes were determined by the frequency domain method using the SLM 4850 MHF Fourier transform lifetime spectrometer (SLM-Aminco, Urbana, Illinois). The fluorescence was excited at $443 \mathrm{~nm}$ wavelength by an intensity-modulated beam of an He:Cd laser. For modulation, the base frequency of $5 \mathrm{MHz}$ and their harmonics up to $250 \mathrm{MHz}$ were simultaneously applied to the light modulator (Pockels cell). The fluorescence response of the sample recorded was subsequently analyzed by fast Fourier transformation. Thus, the phase angle $\Phi$ and demodulation factor $m$ could be obtained for each frequency. The average fluorescence lifetime for each frequency $f$ is given by the equations

$$
\tau_{p}=\omega^{-1} \tan \Phi
$$

and

$$
\tau_{m}=\omega^{-1}\left[\left(1 / m^{2}\right)-1\right]^{1 / 2},
$$

where $\tau_{p}$ is the lifetime determined on the basis of the measured phase angle, $\tau_{m}$ is the lifetime determined from the demodulation factor, $\omega$ equals 2 $\pi f, f$ is the frequency of modulation, and $\Phi$ is the phase angle between exciting and emitted light .

For a single exponential decay, Eqs. (1) and (2) give identical results, i.e., $\tau_{p}=\tau_{m}=\tau$. In heterogeneous samples, $\tau_{p}$ is generally shorter than $\tau_{m}$, which is used as an indicator for the existence of a multiexponential decay. In our experiments we calculated the fluorescence lifetimes from the measured phase angles $\Phi$ because we expected the second lifetime to be below $1 \mathrm{~ns}$. In this case the determination of $\tau_{p}$ is more precise than that of $\tau_{m}{ }^{8}$

\section{RESULTS AND DISCUSSION}

\subsection{ABSORPTION AND CIRCULAR DICHROISM SPECTRA}

Figure 2 shows absorption spectra of POPO- 1 complexed with poly $(\mathrm{dA} \cdot \mathrm{dT})$, poly $(\mathrm{dG} \cdot \mathrm{dC})$ and $\mathrm{ctDNA}$ in comparison with the unbound dye. The measurements were made at a molar ratio $\mathrm{d} / \mathrm{bp}=0.2$ [Figure $2(a)]$ and $d / b p=0.05$ [Figure 2(b)]. For better visualization of the spectral changes, the absorption spectra were normalized. The uncomplexed dye shows maximum absorption at $410 \mathrm{~nm}$ and a shoulder at $428 \mathrm{~nm}$. The occurrence of that shoulder indicates a hydrophobic interaction between the two purple oxazole moieties because it does not exist in the ab- 

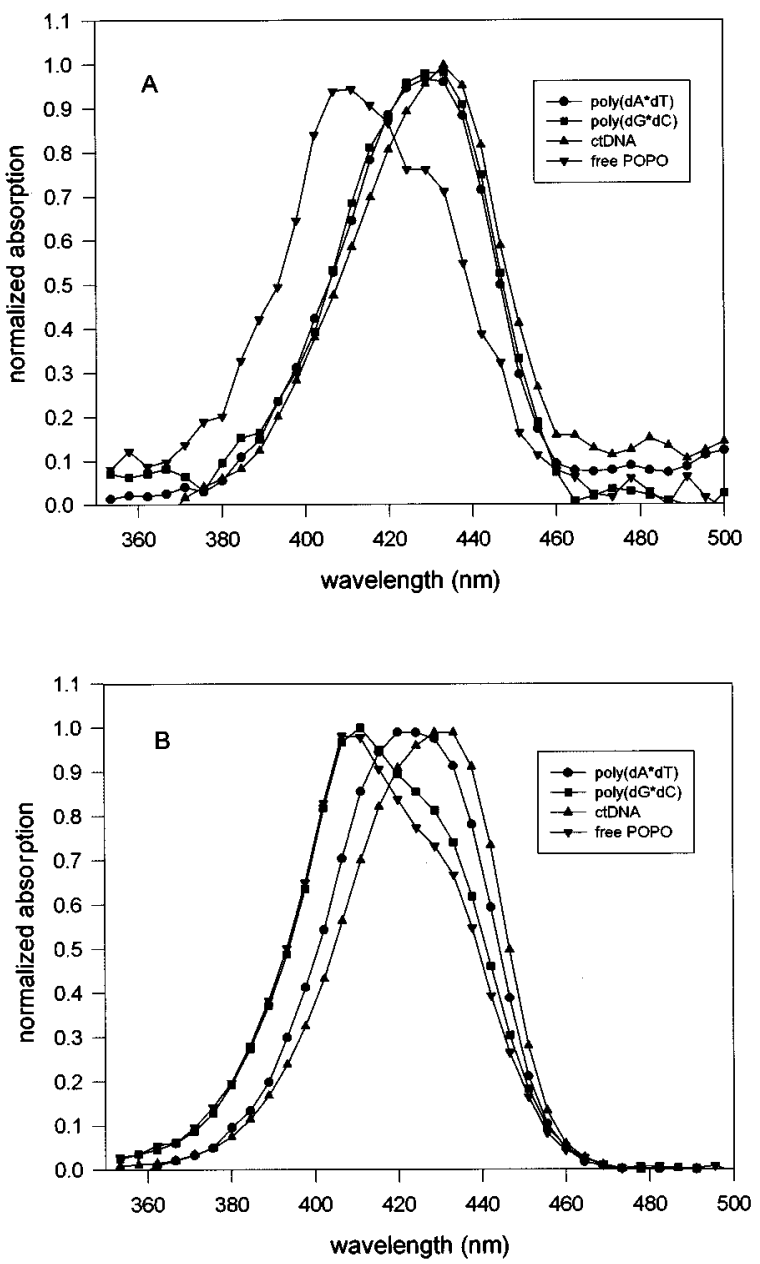

Fig. 2 Absorption spectra (visible region) of POPO-1 bound to polynucleotides at $d / b p=0.05(A)$ and $d / b p=0.2(B)$. The absorption spectra were normalized in order to obtain a better visualization of the spectral shifts that characterize the amount of bound dye.

sorption spectrum of the corresponding monomer PO-PRO-1 (data not shown). ${ }^{4,9}$ Being complexed with polynucleotides, the absorption maximum of POPO-1 is red shifted to $435 \mathrm{~nm}$, which is due to the transfer of the chromophore into a hydrophobic environment. It has been proven by nuclear magnetic resonance (NMR) studies ${ }^{10,11}$ and by flow linear dichroism studies ${ }^{14}$ that intercalation of the oxazole moieties occurs. From the absorption spectra in Figure 2(a), which show similar spectra for all POPO-1/polynucleotide complexes, it can be deduced that at $\mathrm{d} / \mathrm{bp}=0.05$, POPO- 1 is almost completely bound to the polynucleotides, indicating that all dye molecules found appropriate binding sites for intercalation. At $d / b p=0.2$ in the case of poly $(\mathrm{dG} \cdot \mathrm{dC})$ and poly $(\mathrm{dA} \cdot \mathrm{dT})$, part of the dye molecules do not intercalate. This can be seen from their absorption spectra in Figure 2(b), showing absorption maxima that are shifted toward those of free POPO-1. This effect is most pronounced for poly $(\mathrm{dG} \cdot \mathrm{dC})$, indicating an overall smaller affinity

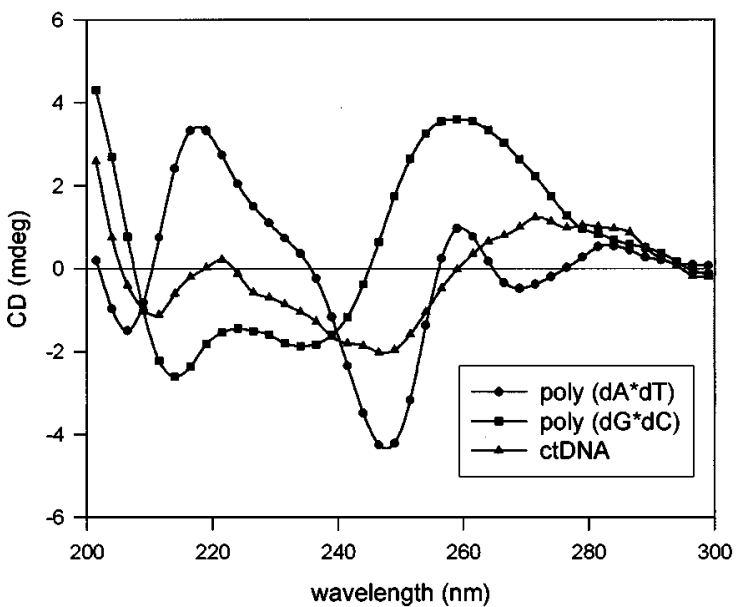

Fig. 3 Circular dichroism spectra of the polynucleotides in $0.15 \mathrm{M}$ $\mathrm{NaCl}$ buffer solution.

of POPO-1 for poly $(\mathrm{dG} \cdot \mathrm{dC})$. An explanation for this can be inferred from the circular dichroism (CD) spectra shown in Figure 3. Poly $(\mathrm{dG} \cdot \mathrm{dC})$ shows a negative circular dichroism at $225 \mathrm{~nm}$ followed by a crossover at $244 \mathrm{~nm}$ and a positive peak at $263 \mathrm{~nm}$, demonstrating that the greater part of the poly $(\mathrm{dG} \cdot \mathrm{dC})$ exists in the A conformation. ${ }^{12}$ According to Gray, Morgan, and Ratliff, ${ }^{12}$ a pure A conformation would have a positive maximum at $254 \mathrm{~nm}$ in the CD spectrum. The shift of the maximum at $254 \mathrm{~nm}$ to $264 \mathrm{~nm}$ shows the parallel existence of poly $(\mathrm{dG} \cdot \mathrm{dC})$ molecules in a $\mathrm{B}$ conformation.

In contrast to poly $(\mathrm{dG} \cdot \mathrm{dC})$, the polynucleotides poly $(\mathrm{dA} \cdot \mathrm{dT})$ and $\mathrm{ctDNA}$ are, under the applied conditions, present preferentially in the $\mathrm{B}$ conformation. It is known from earlier studies ${ }^{13}$ that the $\mathrm{A}$ conformation of DNA inhibits the intercalation of drugs. Thus a large number of nonintercalated POPO- 1 molecules exist in the sample, making the absorption spectra similar to that of the free dye. The detected fluorescence intensity is smaller too because only the POPO-1 molecules that are intercalated into the small fraction of poly $(\mathrm{dG} \cdot \mathrm{dC})$ existing in the B conformation, contribute to the fluorescence.

Owing to electrostatic interaction between the positively charged POPO-1 molecule and the DNA cation, we assume that those dye molecules which are not intercalated do not remain free in the buffer solution. They will be attached to the DNA in unspecific binding modes, as suggested by Larsson, Carlsson, and Jonsson. ${ }^{14}$ We have checked this assumption by fluorescence and fluorescence lifetime studies.

\subsubsection{Fluorescence Intensities}

The fluorescence intensities of POPO- 1 complexed with polynucleotides are shown in Figure 4 for different $d / b p$ ratios in the two buffer systems used. It can be seen in Figure 4(b) that POPO- $1 / \mathrm{ctDNA}$ 

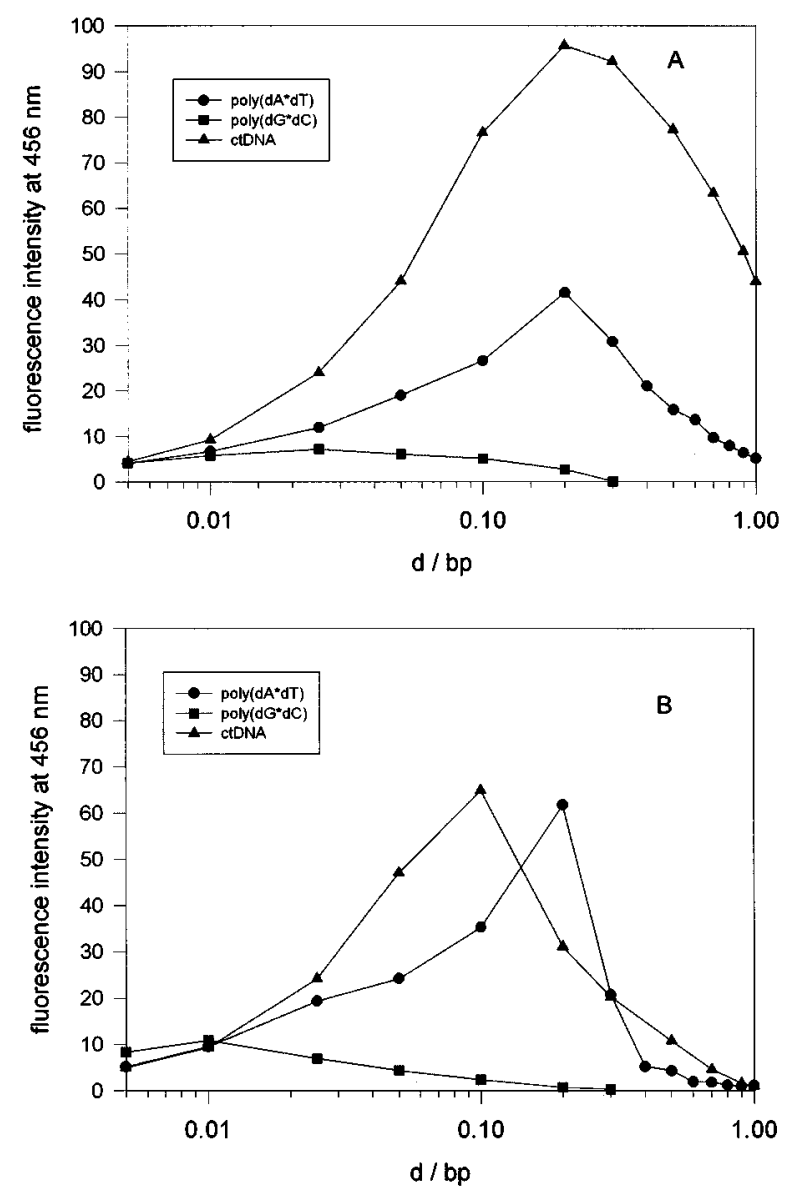

Fig. 4 Fluorescence intensities obtained upon titration of $7.5 \mathrm{nmol}$ of polynucleotide base pairs with POPO-1 in $0.15 \mathrm{M} \mathrm{NaCl}(\mathrm{A})$ and $0.015 \mathrm{M} \mathrm{NaCl}(B)$. Maximum intensity is obtained before the theoretical point of saturation of the polynucleotides with POPO-1 (at $d / b p=0.25$ ) is reached, indicating different types of POPO-1 binding to double-stranded DNA.

complexes show maximum intensity at $\mathrm{d} / \mathrm{bp}=0.1$ in $0.015 \mathrm{M} \mathrm{NaCl}$, while the maximum in $0.15 \mathrm{M} \mathrm{NaCl}$ appears at the higher $\mathrm{d} / \mathrm{bp}=0.2$. This is indicative of the competing effect of sodium ions, which screen the negative charges of the DNA backbone, thus making it less attractive for the positively charged POPO-1 molecules.

Owing to the stepwise addition of the dye solution, all curves first show an increase in the fluorescence intensities of the complexes. One expects for POPO-1, which is virtually nonfluorescent in aqueous solution, an almost constant fluorescence intensity after all the appropriate sites for intercalation are occupied. The strongest fluorescence enhancement is obtained during complexation with ctDNA whereas POPO-1/poly $(\mathrm{dA} \cdot \mathrm{dT})$ complexes show considerably less intensity. This effect speaks for a sequence specificity of POPO-1 binding, which seems to be more complex than a simple AT or GC preference. Indeed, NMR studies yielded a $\left(5^{\prime}-\mathrm{CTAG}-3^{\prime}\right)_{2}$ specificity for the dimer TOTO, which has a molecular structure very similar to
POPO-1. ${ }^{10,15}$ Each titration curve shows a maximum after which further addition of POPO-1 decreases the fluorescence intensity. This quenching effect can be explained by energy transfer processes between the intercalated fluorophors (donor) and those which are bound to the DNA molecule in a different mode (acceptor). Owing to the partial overlap of the fluorescence emission spectra of the intercalated POPO-1 molecules (maximum emission at $456 \mathrm{~nm}$ ) and the absorption spectra of the molecules that are not intercalated (see Figure 2), such an energy transfer is possible. Because the intensity of the energy transfer goes with the sixth power of the distance between donor and acceptor (Förster energy transfer), the rapid decay of the fluorescence after having reached the maximum cannot be explained by an energy transfer to POPO-1 molecules which are free in solution. We are tempted to consider this as another indication for the existence of a second binding mode of POPO-1 to DNA in which the acceptor molecules must have a lower fluorescence quantum yield than the intercalated molecules. This can be elucidated in more detail by fluorescence lifetime spectroscopy.

\subsubsection{Fluorescence Lifetime Studies}

The fluorescence lifetime of a fluorophore is very sensitive to its microenvironment. This makes it possible to distinguish molecules that are bound to the DNA in different modes by their fluorescence lifetime despite the fact that they yield similar fluorescence spectra. Figure 5 shows the fluorescence lifetimes of POPO-1/polynucleotide complexes measured in buffers containing $0.15 \mathrm{M} \mathrm{NaCl}$ [Figure 5(a)] and $0.015 \mathrm{M} \mathrm{NaCl}$ [Figure 5(b)]. The dotted lines interconnect the data points obtained for each titration step for the corresponding POPO-1/ polynucleotide complex. The solid lines represent a curve fitting based on the data points, for better visualization of the trends.

At low mixing ratios $(\mathrm{d} / \mathrm{bp} \leqslant 0.01)$ all POPO- $1 /$ polynucleotide complexes show a single exponential fluorescence decay, indicating a single binding mode. Taking into account the absorption data and the results of NMR studies made by other authors, ${ }^{10,11}$ this fluorescence lifetime arises from the bisintercalated POPO-1 fluorophors. The fluorescence lifetimes vary slightly between 3.6 and 4.0 ns, depending on the actual base pairs at the intercalation sites (see Figure 5).

The phase-modulation data for POPO-1/ poly $(\mathrm{dA} \cdot \mathrm{dT})$ complexes can be fitted by a monoexponential decay law for all titration steps and reveal a decreasing fluorescence lifetime with increasing dye concentration. This is in accord with the decreasing fluorescence intensities of POPO-1/ polynucleotide complexes obtained at higher $\mathrm{d} / \mathrm{bp}$ ratios. The decrease is less pronounced at higher ionic strength of the buffer [Figure 5(a)] in which 

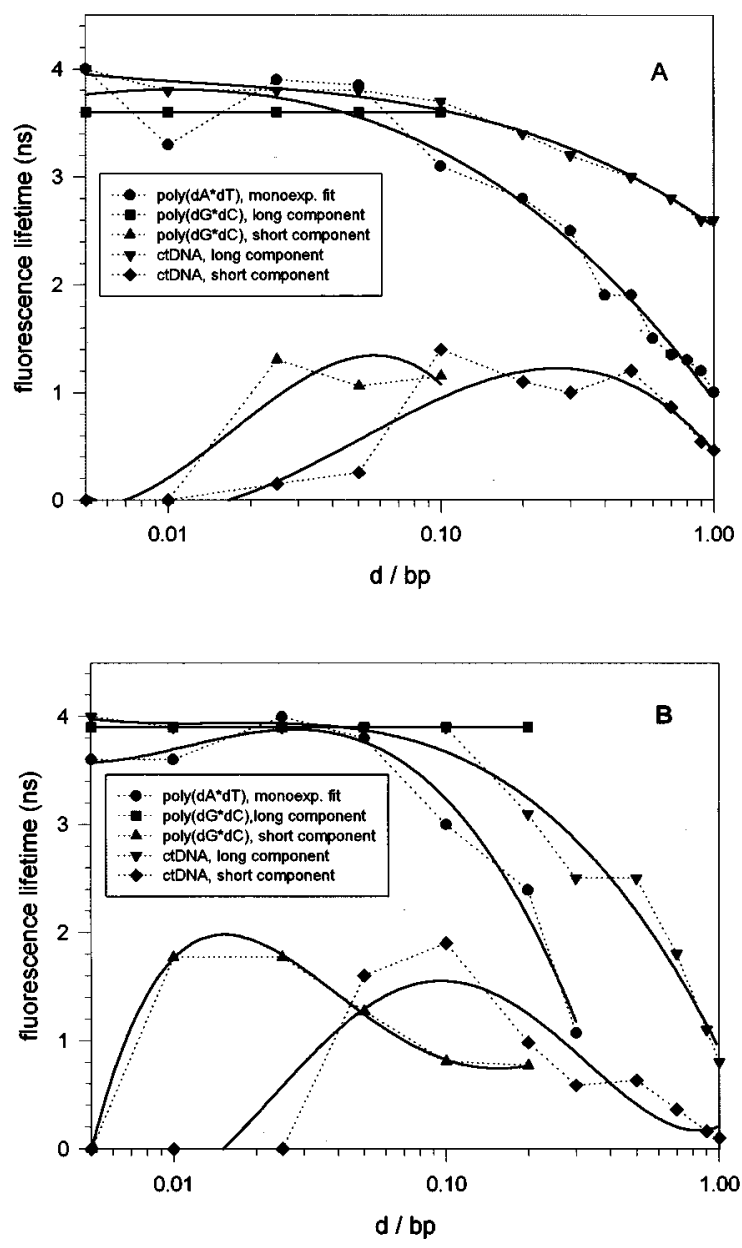

Fig. 5 Fluorescence lifetimes of POPO-1 attached to polynucleotides dependent on the dye-to-base pair ratio (d/bp), measured in $0.15 \mathrm{M} \mathrm{NaCl}(\mathrm{A})$ and $0.015 \mathrm{M} \mathrm{NaCl}(\mathrm{B})$. The dotted lines interconnect the data points measured whereas the bold lines represent a curve fitting for better visualization of the trends.

the charges of the DNA are shielded by the sodium ions.

Intercalation of drugs causes unwinding of the DNA and thus the flexibility of the base pairs increases. ${ }^{16}$ Consequently the rotational mobility around the internuclear bridge between the benzoxazole and the pyridinium rings in the chromophore is increased and the excitation energy can be discharged by nonradiative processes, which results in a shorter lifetime of the excited state. In addition we assume a fluorescence quenching effect due to fluorescence energy transfer between the intercalated POPO-1 fluorophores and those which are electrostatically attached to the polynucleotides after all intercalation sites are occupied. Low ionic strength of the buffer promotes electrostatic attachment of the positively charged POPO-1 molecules, which can be deduced from the more rapidly decaying lifetime of the longer living component in Figure 5(b) in comparison with Figure 5(a).

From the phase-modulation spectra given for POPO-1/ctDNA complexes in Figure 6, it can be

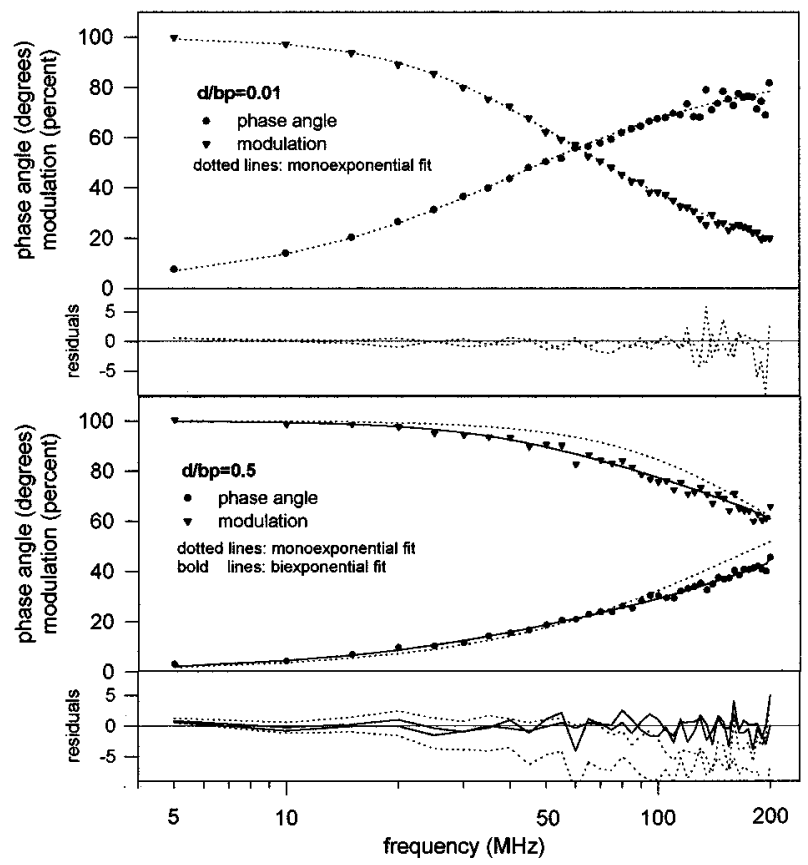

Fig. 6 Example of typical phase-modulation spectra of POPO-1/ ctDNA complexes at $d / b p \leqslant 0.01$ and $d / b p>0.01$. The data were fitted with a monoexponential decay law which, in contrast to $d / b p \leqslant 0.01$, did not give a satisfactory fit for $d / b p>0.01$. A second emitting component having a shorter fluorescence lifetime becomes visible and increases its contribution to the total fluorescence with further increasing $d / b p$. With a biexponential decay law, a good fit of the data is obtained.

seen that starting at $d / b p>0.1$, the phase modulation data of POPO- $1 /$ poly $(\mathrm{dG} \cdot \mathrm{dC})$ and POPO-1/ ctDNA could no longer be fitted by a single exponential decay law. This indicates the occurrence of a second binding mode for POPO-1 arising before all theoretically available intercalation sites are occupied. The second binding mode is characterized by a shorter lifetime varying between $200 \mathrm{ps}$ and $1.2 \mathrm{~ns}$ in $0.15 \mathrm{M} \mathrm{NaCl}$ and $100 \mathrm{ps}$ and $1.8 \mathrm{~ns}$ in $0.015 \mathrm{M}$ $\mathrm{NaCl}$, respectively. We assume the short component to come from POPO-1 molecules in which only one fluorophore of the dimer is intercalated between the base pairs and the other one remains located outside, i.e., sticking into the minor groove of the DNA. The fluorophore located in the minor groove emits with the shorter fluorescence lifetime. The twisted conformation suggested is possibly due to the high flexibility of the fluorophores of the POPO-1 molecule at their bonds to the aliphatic linker. After a steep rise, the lifetime of the second component decreases with increasing $\mathrm{d} / \mathrm{bp}$, similar to the longer component; this is probably caused by changes in the structure of the minor groove which allow the fluorophore a higher rotational mobility. In addition, energy transfer to the electrostatically attached POPO-1 molecules contributes to the overall fluorescence quenching. The lack of a second fluorescence lifetime of POPO-1 complexed with poly $(\mathrm{dA} \cdot \mathrm{dT})$ can be explained by the rather narrow 
and deep minor groove in poly $(\mathrm{dA} \cdot \mathrm{dT}),{ }^{17}$ which allows the chromophore only a small rotational mobility or none. Thus its fluorescence lifetime becomes very close to that of intercalated fluorophors and cannot be detected by the measurements.

\section{CONCLUSIONS}

The interaction of the dimer POPO- 1 with polynucleotides is a complex process of bisintercalation and monointercalation in connection with minor groove binding as well as electrostatic attachment. Bisintercalation can only be obtained at a high excess of polynucleotides, i.e., mixing ratios of $\mathrm{d} / \mathrm{bp} \leqslant 0.01$. Increasing the amount of dye added, leads to a parallel existence of different binding modes, i.e., bisintercalated molecules and those in which only one of the two fluorophores is intercalated and the other one remains in the minor groove (monointercalated dimer molecules). After saturation of the polynucleotide with POPO-1, which theoretically occurs at $d / b p=0.25$, but in practice depends on the ionic strength of the buffer and the polynucleotide itself, the POPO- 1 molecules added are electrostatically attached to the DNA and sustain their rotational motion. Thus their fluorescence becomes quenched, making them very similar to free POPO- 1 molecules in aqueous solution.

In fluorescence intensity measurements of POPO-1 dissolved in organic solvents (data not shown), only a moderate fluorescence enhancement of POPO- 1 was obtained, even in solvents with low dielectric constants. Thus the fluorescence enhancement must be explained by two different processes. The first is the restricted rotational motion of the chromophore in the intercalation pocket, as already suggested by other authors for related dyes ${ }^{4,10}$ and the second, hence with a much smaller contribution, is the hydrophobic environment between the base pairs.

The intercalation of many dye molecules causes structural changes of the DNA molecule such as unwinding and increased mobility of the base pairs, thus decreasing the fluorescence lifetime of the intercalated fluorophores by allowing them a higher rotational mobility. In addition, the minor groove becomes wider, which in consequence leads to a quenching of the POPO-1 molecules bound there. Thus the overall decreasing fluorescence intensity at high $d / b p$ is caused by various mechanisms.

For possible clinical applications, it should be taken into account that only the bisintercalated POPO-1 molecules show the high binding affinity that is required for inhibiting enzyme activity. Thus a low dose application seems to be sufficient for affecting the enzyme activity during DNA replication. Hence, due to their charge, POPO-1 molecules probably will not penetrate the cell membrane and thus should be injected directly into the cell.

\section{Acknowledgments}

We would like to thank Mrs. Gabriele Guenther for carefully performing the titration experiments and Mr. Steffen Kirschstein for measuring the CD spectra.

\section{REFERENCES}

1. H. S. Rye, J. M. Dabora, M. A. Quesada, R. A. Mathies, and A. N. Glazer, "Fluorimetric assay using dimeric dyes for double and single stranded DNA and RNA with picogram sensitivity," Anal. Biochem. 208, 144-150 (1993).

2. B. Gaugin, J. Barbet, R. Oberlin, B. P. Roques, and J. B. Le Pecq, "DNA bifunctional intercalators. 2. Fluorescence properties and DNA binding interaction of an ethidium homodimer and an acridine ethidium heterodimer," Biochemistry 17, 5078-5088 (1978).

3. A. N. Glazer and H. S. Rye, "Stable dye-DNA intercalation complexes as reagents for high-sensitivity fluorescence detection," Nature 217, 414-431 (1992).

4. C. Carlsson, A. Larsson, M. Jonsson, B. Albinsson, and B. Norden, "Optical and photophysical properties of the Oxazole Yellow DNA probes YO and YOYO," J. Phys. Chem. 98, 10313-10321 (1994).

5. L. M. Popa, S. Winter, and G. Löber, "Some new properties of DNA-YOYO-3 homodimer complexes revealed by electrophoresis and fluorescence lifetime measurements," Biochem. Molec. Biol. Internat. 34, 1189-1196 (1994).

6. B. Akerman and E. Tuite, "Single- and double-strand photocleavage of DNA by YOYO and TOTO," Nucleic Acid Res. 24, 1080-1090 (1996).

7. A. Larsson, C. Carlsson, M. Johnsson, and B. Albinsson, "Characterization of the fluorescent dyes YO and YOYO to DNA by polarized light spectroscopy," J. Am. Chem. Soc. 116, 8459-8465 (1994).

8. J. R. Lakowicz, Principles of Fluorescence Spectroscopy, Plenum Press, New York (1983).

9. T. L. Netzel, K. Nafisi, M. Zhao, J. R. Lenhard, and T. Johnson, "Base-content dependence of emission enhancements, quantum yields and lifetimes for cyanine dyes bound to double-strand DNA: photophysical properties of monomeric and bichromophoric DNA stains," J. Phys. Chem. 99, 17936-17947 (1995).

10. H. P. Spielmann, D. E. Wemmer, and J. P. Jacobsen, "Solution structure of a DNA complex with the fluorescent bisintercalator TOTO determined by NMR spectroscopy," Biochemistry 34, 8542-8553 (1995).

11. L. F. Hansen, L. K. Jensen, and J. P. Jacobsen, "Bisintercalation of a homodimeric thiazole orange dye in symmetrical pyrimidine-pyrimidine-purine-purine oligonucleotides," Nucleic Acid Res. 5, 859-867 (1996).

12. D. M. Gray, A. R. Morgan, and R. L. Ratliff, "A comparison of the circular dichroism spectra of the homopurinehomopyrimidine and mixed purine-pyrimidine types," Nucleic Acid Res. 5, 3679-3695 (1978).

13. W. Guschlbauer, "Polynucleotides" in Encyclopedia of Polymer Science and Engineering, Vol. 12, Wiley, New York (1988).

14. A. Larsson, C. Carlsson, and M. Jonsson, "Characterization of the binding of YO to $[\mathrm{Poly}(\mathrm{dA}-\mathrm{dT})]_{2}$ and $[\mathrm{Poly}(\mathrm{dG}-\mathrm{dC})]_{2}$ and of the fluorescent properties of $\mathrm{YO}$ and YOYO complexed with the polynucleotides and double-stranded DNA," Biopolymers 36, 153-167 (1995).

15. J. P. Jacobsen, J. B. Pedersen, L. F. Hansen, and D. E. Wemmer, "Site selective intercalation of a homodimeric thiazole orange dye in DNA oligonucleotides," Nucleic Acid Res. 23, 753-760 (1995).

16. P. Herzyk, S. Neidle, and J. M. Goodfellow, "Conformation and dynamics of drug-DNA intercalation," J. Biomolec. Structure Dynamics 10(1), 97-139 (1992).

17. C. V. Kumar, R. S. Turner, and E. H. Asuncion, "Groove binding of a styrylcyanine dye to the DNA double helix: the salt effect,"' J. Photochem. Photobiol. 74, 231-238 (1993). 